\title{
Effects of Different Types of Verbal Activities on Heart Rate Variability
}

\author{
Ping Shi, Youfang Fang, Hongliu Yu ${ }^{*}$ \\ School of Medical Instrument and Food Engineering, University of Shanghai for Science and Technology, Shanghai, China \\ Email: "garendon@163.com \\ Received July 2013
}

\begin{abstract}
In the present study, the effects of different types of verbal activities on heart rate variability (HRV) were investigated. ECG signals were recorded in ten volunteers during resting (R), reading silently (RS), reading aloud (RA) and talking freely (TF). Time domain, frequency domain and Poincaré plot measures of HRV were calculated for analyzing. Time domain parameter of pNN50, frequency domain parameter of LF (n.u.) and Poincaré plot parameter of SD1/SD2 were found statistically difference in RA and TF compared to R and RS. The results in this study show that HRV decreased while subjects were reading aloud and talking freely. The results also indicated that verbal activities of reading aloud and talking freely improve the sympathetic nervous activity.
\end{abstract}

Keywords: Verbal Activities; Heart Rate Variability; Time Domain Analysis; Frequency Domain Analysis; Poincaré Plot Parameters

\section{Introduction}

Heart rate variability (HRV) has been recognized as a simple and valuable tool to assess the regulation of heart rate behavior. It reflects the homeostatic interplay between perturbations in cardiovascular functions and the dynamic responses of the cardiovascular regulatory systems [1]. There is considerable interest in HRV because their measures such as the standard deviation of the interbeat intervals (the RRI), have been shown to be some of the strongest independent predictors of mortality after myocardial infarction [2]. Moreover, other techniques such as spectral analysis and nonlinear analysis (such as Poincaré plot) of the RRI of HR have been widely used in HRV studies [3-5], and on some occasions they have been shown to discriminate between subjects with different cardiac conditions as well as to predict mortality in some groups of patients. Several types of verbalization including reading silently, reading aloud and talking freely occurs in our daily life. The aim of this paper is to investigate the effect of different conditions of verbalization on HRV.

\section{Methods}

\subsection{Protocols}

The study was performed in ten normotensive male volunteers (20.2 \pm 1.6 years). None of the subjects was

*Corresponding author. taking medication. The subjects gave written informed consent and the study protocol was approved by the Ethics Committee of the University of Shanghai for Science and Technology, China.

All the tests were carried out while the subjects rested in the 135 degree sitting position on a comfortable chair. Subjects were asked a resting period of at least $15 \mathrm{mi}-$ nutes before ECG signals were recording. Recordings were made in random order during: 1$)$ resting; 2 ) reading a text silently (5 min); 3) reading the same text aloud (5 min); 4) normal talking (5 min).

\subsection{Measurement of $\mathrm{HRV}$}

Like many other physiological parameters, HRV is influenced by a variety of factors like sex, age, diurnal rhythms, respiration, fitness levels, posture and physical activity. In 1996, Task Force of the European Society of Cardiology (ESC) and the North American Society of Pacing and Electrophysiology (NASPE) defined and established standards of measurement, physiological interpretation and clinical use of HRV [1].

The time domain measures of HRV are based on statistical or geometric analyses of the lengths of intervals between successive normal complexes. Frequency domain HRV indices are determined by calculating the power spectral density of the ECG recording using a nonparametric fast Fourier transform (FFT) algorithm. Poincaré plot as a nonlinear method is a scatter outline of the 
current R-R interval plotted against the preceding R-R interval. The plot delivers not only an outline but also a detail of beat-to-beat behavior of cardio-physiology. Time domain parameters, frequency domain parameters and Poincaré plot parameters included in this paper for evaluation were listed in Table 1.

\subsection{Statistics}

Values are presented as mean \pm standard error of the mean (SEM). The significance of difference was analyzed using one-way ANOVA with repeated-measures. The statistical analyses were run in MATLAB software (MathWorks Inc., MA, USA). A P $<0.05$ was considered statistically significant.

\section{Results}

In this paper, the parameters of time domain, frequent domain and Poincaré plot parameters were sued to evaluate the HRV in different types of verbal activities. As shown in Figure 1, pNN50 in RA and TF significantly decreased compared to $\mathrm{R}$ and $\mathrm{RS}(\mathrm{P}<0.05)$. SDRR in TF significantly increased compared to $\mathrm{R}$ and $\mathrm{RS}(\mathrm{P}<0.05)$. Smaller RRIs were found in RA and TF compared to R and RS although on significant differences were found. No differences were found for RMSSD between different types of verbal activities.

Table 1. Time domain parameters, frequency domain parameters and Poincaré plot parameters included in this paper for evaluation.

\begin{tabular}{|c|c|c|}
\hline Variable & Units & Description \\
\hline \multicolumn{3}{|c|}{ Time domain parameters of $\mathrm{HRV}$} \\
\hline RRI & ms & The length of R-wave to R-wave intervals. \\
\hline SDNN & ms & Standard deviation of all NN intervals. \\
\hline RMSSD & ms & $\begin{array}{l}\text { The square root of the mean of the sum of } \\
\text { the squares of differences between adjacent } \\
\text { NN intervals. }\end{array}$ \\
\hline pNN50 & $\%$ & $\begin{array}{l}\text { NN50 count divided by the total number } \\
\text { of all NN intervals. }\end{array}$ \\
\hline \multicolumn{3}{|c|}{ Frequency domain parameters of HRV } \\
\hline LF norm & n.u. & $\begin{array}{l}\text { LF power in normalized units, } \\
\text { LF/(Total Power-VLF) } \times 100 \text {, } \\
\text { Frequency range: } 0.04-0.15 \mathrm{~Hz}\end{array}$ \\
\hline HF norm & n.u. & $\begin{array}{l}\text { HF power in normalized units, } \\
\text { HF/(Total Power-VLF) } \times 100 \text {, } \\
\text { Frequency range: } 0.15-0.4 \mathrm{~Hz}\end{array}$ \\
\hline $\mathrm{LF} / \mathrm{HF}$ & & Ratio LF $\left[\mathrm{ms}^{2}\right] / \mathrm{HF}\left[\mathrm{ms}^{2}\right]$ \\
\hline \multicolumn{3}{|c|}{ Poincaré plot parameters of HRV } \\
\hline SD1 & $\mathrm{ms}$ & $\begin{array}{l}\text { instantaneous beat-to-beat variance } \\
\text { of the R-R intervals }\end{array}$ \\
\hline SD2 & ms & $\begin{array}{l}\text { the long-term continuous variance } \\
\text { of all R-R intervals }\end{array}$ \\
\hline SD1/SD2 & & Ratio SDl/SD2 \\
\hline
\end{tabular}

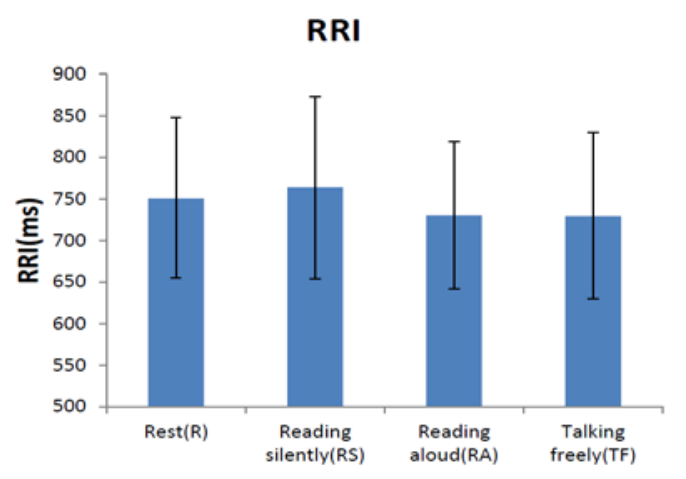

(a)

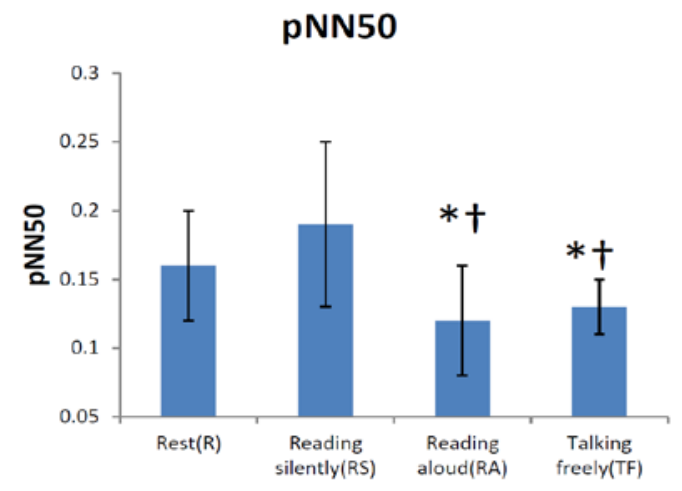

(b)

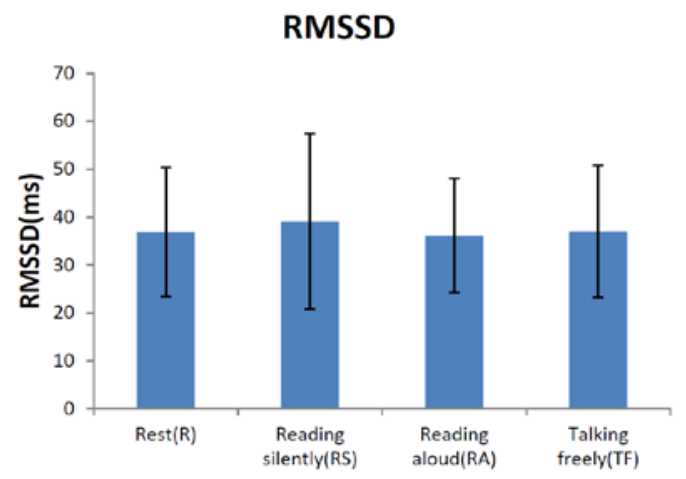

(c)

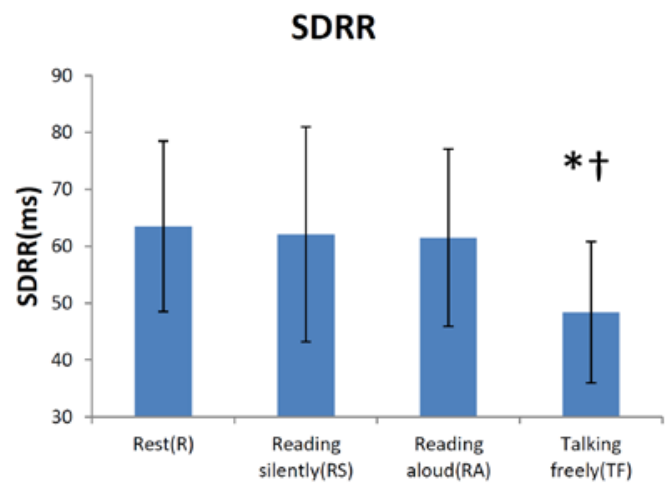

(d)

Figure 1. Time domain parameters of HRV for different types of verbal activities. (a) RRI, (b) pNN50, (c) RMSSD, (d) SDRR * $p<0.05$ compared to $R$, $\nmid p<0.05$ compared to RS. 
As shown in Figure 2, RA and TF augmented LF (n.u.) significantly compared to R and RS ( $\mathrm{P}<0.05)$. LF/HF increased significantly in TF compared to $\mathrm{R}(\mathrm{P}<0.05)$. No differences were found for HF (n.u.) between different types of verbal activities.

As shown in Figure 3, SD2 significantly increased in TF compared to R and RS, and significantly increased in RA compared to RS. Significant smaller SD1/SDs were found in RA and TF compared to R and RS ( $<<0.05)$. No differences were found for SD2 between different types of verbal activities.

\section{LF(n.u.)}

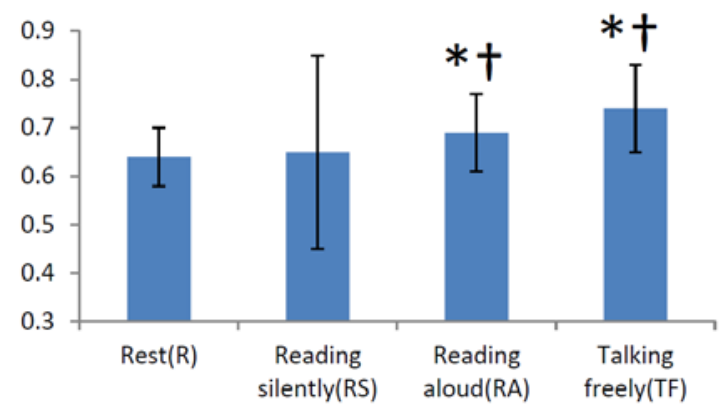

(a)

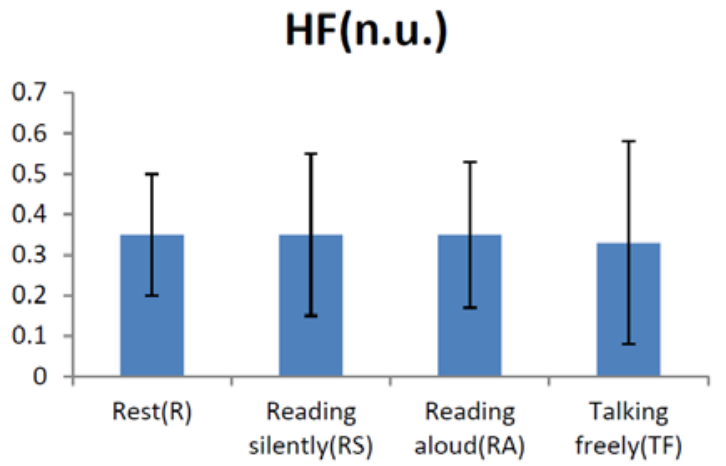

(b)

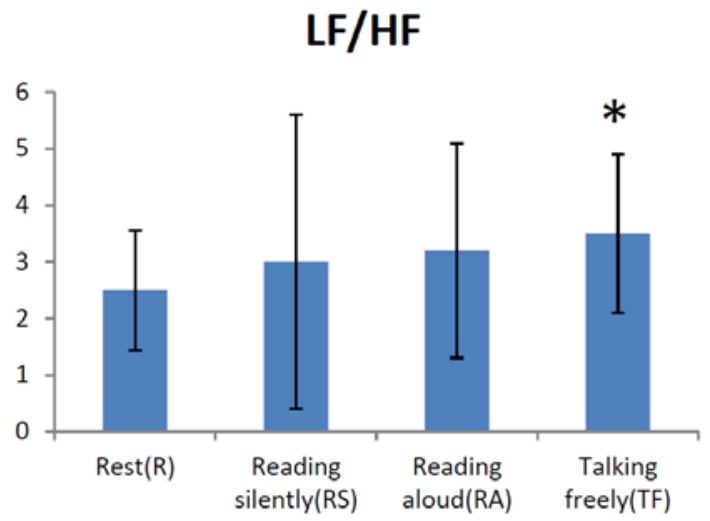

(c)

Figure 2. Frequency domain parameters of HRV for different types of verbal activities. (a) LF (n.u.), (b) HF (n.u.), (c) LF/HF. * $p<0.05$ compared to $R, \dagger p<0.05$ compared to RS.

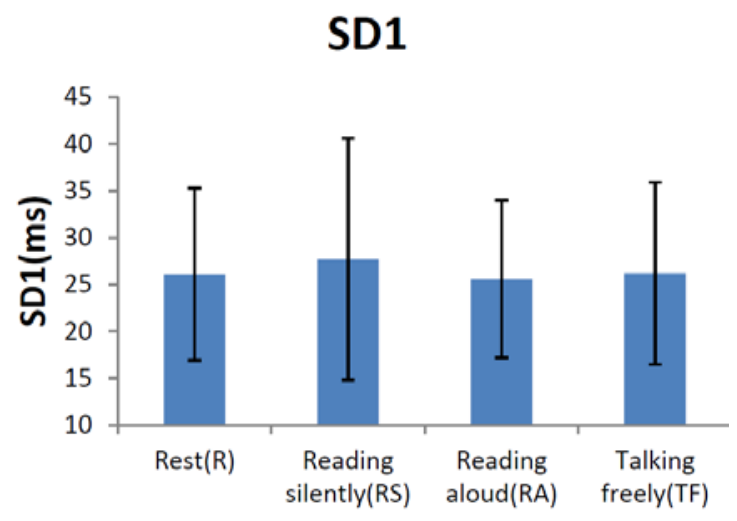

(a)

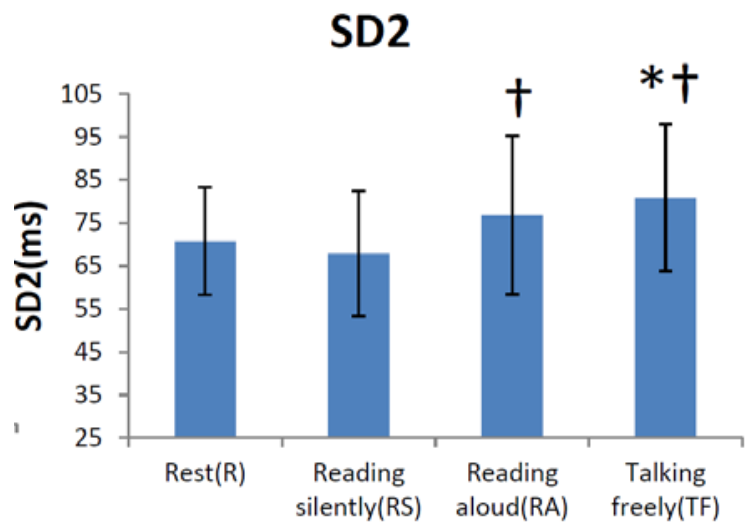

(b)

SD1/SD2

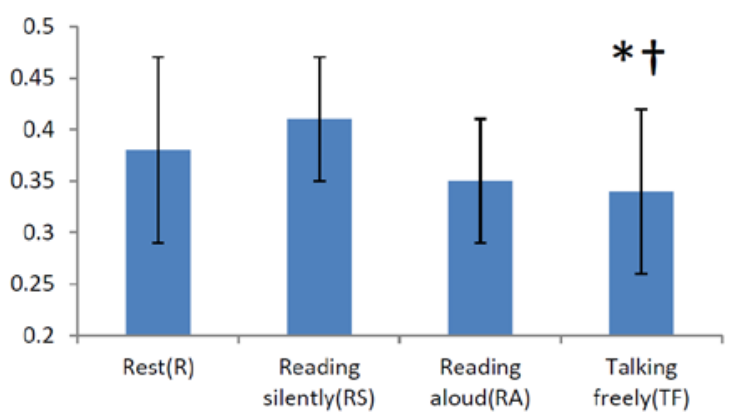

(c)

Figure 3. Poincaré plot parameters of $\mathrm{HRV}$ for different types of verbal activities. (a) SD1, (b) SD2, (3) SD1/SD2. *p $<0.05$ compared to $\mathrm{R}, \boldsymbol{\dagger p}<\mathbf{0 . 0 5}$ compared to $\mathrm{RS}$.

\section{Discussion}

HRV, the amount of fluctuation of the beat to beat differences, is known to be a reliable, noninvasive marker of autonomic nervous system activity. Assessment of HRV may provide quantitative information on the modulation of cardiac vagal and sympathetic nerve input. HRV analysis is a recognized tool for the estimation of cardiac autonomic modulations. Reduced HRV is a powerful and independent predictor of an adverse prognosis in patients with cardiac disease. In this study, the meas- 
ures of HRV include time domain parameters, frequency domain parameters and Poincaré plot parameters.

Both reading aloud and talking freely caused the decreased pNN50s, indicating a reduction in HRV. The condition of talking freely tended to a higher sympathetic activity as evidenced by changes of SDNN only found in talking freely.

Spectral analysis of HRV is a useful, noninvasive technique to study the short-term (2 - $5 \mathrm{~min}$ ) autonomic modulation of HR. For the frequency-domain parameters of HRV, the power value of the HF content is considered a pure measure of parasympathetic activity, while the power value of the LF content is reflective of both sympathetic modulation and parasympathetic tone $[1,6]$. $\mathrm{LF} / \mathrm{HF}$ ratio was considered as an index of sympathetic activity as well as the balance between the sympathetic and parasympathetic nerves [6-8]. The results, from spectral analysis of HRV in this study, indicated that the conditions of reading aloud and free talking influence the sympathetic modulation and parasympathetic tone, and that sympathetic activity increased only during free talking compared to the condition of resting.

In this study, Poincaré plot parameters were investigated to reflect autonomic function changes associated with different types of verbal activities. SD1 is mediated by vagal efferent activity. SD2 is influenced by both parasympathetic and sympathetic tone. SD1/SD2 ratio could be used as an indicator of sympathetic activity. The results from Poincaré plot analysis of HRV in this study indicated that the sympathetic activity increased in conditions of reading aloud and free talking compared to resting and reading silently.

\section{Conclusion}

Overall, these results indicated that HRV decreased while subjects were reading aloud and talking freely, and that verbal activities of reading aloud and talking freely improved the sympathetic nervous activity. Although both sympathetic and parasympathetic activity were modulated evidenced by the present results, the parasympathetic activity cannot be determined by the present results.

\section{REFERENCES}

[1] Task Force of the European Society of Cardiology and the North American Society of Pacing and Electrophysiology, "Heart Rate Variability: Standards of Measurement, Physiological Interpretation and Clinical Use.” Circulation, Vol. 93, No. 5, 1996, pp. 1043-1065. http://dx.doi.org/10.1161/01.CIR.93.5.1043

[2] C. S. Zuern, P. Barthel and A. Bauer, "Heart Rate Turbulence as Risk-Predictor after Myocardial Infarction,” Frontiers in Physiology, Vol. 2, 2011, p. 99. http://dx.doi.org/10.3389/fphys.2011.00099

[3] D. Chamchad, J. C. Horrow, L. Nakhamchik and V. A. Arkoosh, "Heart Rate Variability Changes during Pregnancy: An Observational Study,” International Journal of Obstetric Anesthesia, Vol. 16, No. 2, 2007, pp. 106-109 2007. http://dx.doi.org/10.1016/j.ijoa.2006.08.008

[4] H. V. Huikuri, A. M. Poutiainen, T. H. Mäkikallio, M. J. Koistinen, K. E. Airaksinen, R. D. Mitrani, R. J. Myerburg and A. Castellanos, "Dynamic Behavior and Autonomic Regulation of Ectopic Atrial Pacemakers," Circulation, Vol. 100, No. 13, 1999, pp. 1416-1422. http://dx.doi.org/10.1161/01.CIR.100.13.1416

[5] M. P. Tulppo, T. H. Mäkikallio, T. E. Takala, T. Seppänen and H. V. Huikuri, "Quantitative Beat-to-Beat Analysis of Heart Rate Dynamics during Exercise,” American Journal of Physiology, Vol. 271, No. 1, 1996, pp. H244-H252.

[6] A. Malliani, M. Pagani, F. Lombardi and S. Cerutti, "Cardiovascular Neural Regulation Explored in the Frequency Domain,” Circulation, Vol. 84, No. 1, 1991, pp. 482-492. http://dx.doi.org/10.1161/01.CIR.84.2.482

[7] M. Pagani, F. Lombardi, S. Guzzetti, O. Rimoldi, R. Furlan, P. Pizzinelli, G. Sandrone, G. Malfatto, S. Dell’Orto, E. Piccaluga, et al., "Power Spectral Analysis of Heart Rate and Arterial Pressure Variabilities as a Marker of Sympatho-Vagal Interaction in Man and Conscious Dog," Circulation Research, Vol. 59, No. 2, 1986, pp. 178-193. http://dx.doi.org/10.1161/01.RES.59.2.178

[8] D. L. Eckberg, "Sympathovagal Balance: A Critical Appraisal,” Circulation, Vol. 96, No. 9, 1997, pp. 3224-3232. http://dx.doi.org/10.1161/01.CIR.96.9.3224 